УДК 339.371

ПЕРСПЕКТИВЫ И ПРОБЛЕМЫ РОБОТИЗАЦИИ ДОСТАВКИ ПОСЛЕДНЕЙ МИЛИ. АКТУАЛЬНОСТЬ ЗАДАЧИ И КОНЦЕПЦИИ РЕШЕНИЯ. ЧАСТЬ І. \author{
DOI: $10.31618 /$ ESU.2413-9335.2021.2.92.1516 \\ Хамуков Ю.Х. \\ ФГБУН Институт информатики и проблем регионального управления \\ Кабардино-Балкарского научного иентра РАН \\ 360000, КБР, г. Нальчик, ул. И. Арманд, 37-а
}

Канокова М.A.

Федеральное государственное бюджетное научное учреждение «Федеральный научный иентр «Кабардино-Балкарский научный иентр Российской академии наук», 360003, Россия, Кабардино-Балкарская Республика, г. Нальчик, ул. И. Арманд, 37а.

\title{
PROSPECTS AND PROBLEMS OF ROBOTIC DELIVERY OF THE LAST MILE. THE RELEVANCE OF THE PROBLEM AND THE CONCEPT OF THE SOLUTION. PART I.
}

\author{
Yuri Khabijevich Khamukov \\ PhD, Head of Department "Multi-agent systems" of the Institute of Informatics and Regional Management \\ Problems - Branch of the Federal State Budgetary Institution "Federal Research Center" \\ Kabardino-Balkaria Scientific Center of the Russian Academy of Sciences ", \\ 360003, Russia, Kabardino-Balkaria Republic, Nalchik, ul. I. Armand, 37a Phone.:+7(967)415-20-17 \\ Madina Alikovna Kanokova \\ Head of the Laboratory "Development of Modes for the Promotion of Innovative Projects", \\ Federal State Budgetary Scientific Establishment "Federal Scientific Center "Kabardin-Balkar Scientific \\ Center of the Russian Academy of Sciences" \\ 360000, Nalchik, 37-a, I. Armand street \\ Phone.: +7 (928) 077-70-92
}

\begin{abstract}
ABSTARCT
Рынок экспресс-доставки в последние годы растет на уровне 3-4\%, и даже в этих условиях не только не насыщается, но спрос на нём возрастает. По данным Oxford Economics, pост рынка грузовых авиаперевозок, определяющий объем рынка экспресс-доставки, в период с 2013 по 2018 год ускорялся временами до 7\% в год [1].

Наибольшие изменения произошли в 2016-17 годы вследствие технологического прорыва в области логистики с внедрением сервисов «доставка дронами», «обработка заказов на блокчейне», «расчет режима доставки с помощью искусственного интеллекта» и др. Ожидалось, что, в связи с ростом спроса на услуги быстрой гарантированной доставки, количество сотрудников в сфере экспресс-доставки во всем мире вырастет до 4,5 миллионов в течение следующих нескольких лет. Но пандемия коронавируса ускорила этот процесс.

В исследовании «Будущее грузовых перевозок. Как новые технологии и новое мышление могут изменить движение товаров», представленное международной сетью консалтинговых компаний Deloitte в 2017 году, констатируется, что перевозчики уже решили многие проблемы, связанные с транспортировкой грузов. Но этап «доставки последней мили» (last mile delivery) остался лимитирующим развитие услуги доставки. На этом этапе возникают убытки компаний из-за концентрации логистических, алгоритмических и кинематических задач, которые невозможно автоматизировать современными средствами и технологиями замещения человеческого труда.

Следовательно, использование альтернативных, нетрадиционных технологий на данном этапе является ключевым условием массового развития доставки.

\section{АННОТАЦИЯ}

The express delivery market in recent years has been growing at the level of 3-4\%, and even in these conditions, not only is it not saturated, but the demand for it is growing. According to Oxford Economics, the growth of the air cargo market, which determines the volume of the express delivery market, accelerated at times up to $7 \%$ per year from 2013 to 2018 [1].

The biggest changes took place in 2016-17 due to a technological breakthrough in the field of logistics with the introduction of services such as drone delivery, processing orders on the blockchain, calculation of the delivery mode using artificial intelligence, etc. It was expected that due to the growing demand on fast delivery guaranteed, the number of express delivery employees worldwide will grow to 4.5 million over the next few years. But the coronavirus pandemic has accelerated this process.

In the study "The Future of Freight Transportation. How new technologies and new thinking can change the movement of goods", presented by the international network of consulting companies Deloitte in 2017, states that carriers have already solved many of the problems associated with the transportation of goods. But the "last mile delivery" stage has remained limiting the development of the delivery service. At this stage, companies suffer
\end{abstract}


losses due to the concentration of logistics, algorithmic and kinematic tasks that cannot be automated with modern means and technologies for replacing human labor.

Consequently, the use of alternative, unconventional technologies at this stage is a key condition for the mass development of delivery.

Ключевые слова: курьер, доставка, ритейл, е-коммерция, онлайн-торговля, потребности, спрос, безлюдность.

Keywords: courier, delivery, retail, e-commerce, online commerce, needs, demand, desertion.

Введение. В публикациях по доставке последней мили применяется термин «курьер» от французского courrier - «бегун» и латинского currere - «бегать, быстро двигаться». В современном употреблении термин обозначает человека или компанию, нанимаемую для неотложной доставки сообщений, писем, иной корреспонденции, а также небольших грузов ${ }^{1}$.

Спрос на услугу, называемую доставкой последней мили, возник и неуклонно рос с античных, а вероятнее всего - с гораздо более ранних времён, с момента появления разделения труда и имущественного расслоения раннего общества. В античном Риме курьерская доставка стала прекрасно оплачиваемой и поддерживаемой государством системой безостановочного движения доставщиков указов, новостей и различных сообщений, и посылок. С несущественными изменениями структуры, спрос на скорую доставку продолжал расширяться в средние века, применяясь на всех уровнях организации жизнедеятельности общества от обмена срочными посылками между лавками и мастерскими до официальной государственной курьерской службы.

В данном исследовании понятие доставки трактуется как процесс транспортировки товаров из исходного местоположения в заранее определенное место назначения в виде оказания единичных услуг с обеспечением своевременности и безопасности, предоставлением отчета о доставке в виде отметки о вручении, соблюдения специализации и индивидуальности услуг. При этом доставка последней мили отличается от доставки ежедневной почты тем, что последняя имеет свою специализированную инфраструктуру, обеспечивающую услугу дешево и своевременно при всевозможных обстоятельствах. Услуги курьеров-доставщиков последней мили многообразней доставки ежедневной почты и существенно дороже из-за персонификации услуг в

1 Электронная энциклопедия «Академик». Эл. ресурс: https://dic.academic.ru/dic.nsf/ruwiki/99690

2 Доставка Последней Мили В 2021 Году: Руководство По Пониманию Программного Обеспечения Последней Мили И Его Влияния // Логистическая платформа FarEye. [Электронный pecypc]. https://www.getfareye.com/insights/blog/rukovodstvo-podostavke-posledney-mili

${ }^{3}$ Гайд по UX-проблемам // Интернет-издание о бизнесе, стартапах, инновациях, маркетинге и технологиях Vc.ru. июнь 2021. Эл. pecypc: https://vc.ru/services/260024-gaydpo-ux-problemam

4 Робот-курьер Serve доставит вам еду на дом // Международная группа национальных ритейлеров соответствии с требованиями современных рыночных трендов.

В работе представлены результаты поиска ответов на следующие вопросы о сущности задачи, решаемой при разработке концепции роботизации курьерской доставки и технического решения доставки «последней мили»:

1. Чем обусловлена актуальность доставки «последней мили»?

2. Какие задачи решает доставка «последней мили»?

3. Что включает процесс доставки «последней мили»?

4. Какие проблемы связаны с осуществлением доставки «последней мили»?

5. В чём заключается проблема отслеживания доставки «последней мили»?

6. Какие известны технологии и тренды развития доставки «последней мили»?

7. Какие преимущества предоставляет решение задачи безлюдной доставки «последней мили»?

8. В чём выражаются особенности программного обеспечения автоматических систем для осуществления Last Mile Delivery?

9. Какие решения задачи доставки «последней мили» известны в настоящее время?

Формат изложения результатов исследования аналогичен формату руководства ${ }^{2}$.

Актуальность и исходные положения исследования. Доставка товаров - один из ключевых сервисов онлайн-торговли и, соответственно, предмет растущей дискуссии ${ }^{3} 456$ $[2,3,4]$.

Опираясь на результаты своих исследований выбора ритейлера покупателями, международная консалтинговая сеть компаний PricewaterhouseCoopers (PwC) определила, что, главным фактором - после цены - является наличие доставки. Вторым фактором является скорость и своевременность доставки. Исходя из этих

Euronics.

Эл.

pecypc: https://www.sulpak.kz/Article/6562/robot_kurer_serve_dost avit_vam_edu_na_dom

5 Бойко А. Уличные роботы-курьеры // Электронный журнал RoboTrends. Эл. ресурс: http://robotrends.ru/robopedia/ulichnye-roboty-kurery

6 В Британии десятки роботов-курьеров доставляют сотни тысяч заказов // Российский интернет-портал и аналитическое агентство TAdviser. 2020. Эл. ресурс: https://www.tadviser.ru/index.php/\%D0\%9A\%D0\%BE\%D0 \%BC\%D0\%BF\%D0\%B0\%D0\%BD\%D0\%B8\%D1\%8F:Sta rship 
результатов, компания прогнозировала рост рынка Last Mile Delivery в 2020-м году до 127 миллиардов USD.

Bce прочие тренды в ритейле, если непосредственно не подтверждали подобные прогнозы, то, во всяком случае, не противоречили им. В мегаполисах доставка оценивается выше, чем в регионах. Доставкой потребительских товаров пользуются большие когорты населения - уже в 2017 году пользователи смартфонов в возрасте от 25 до 44 лет догнали по числу заказов 18-летних. Постепенно подтягивается и старшая аудитория. По данным международной сети консалтинговых компаний $\mathrm{PwC}$, чаще всего в интернет-магазинах заказывают люди от 25 до 34 лет. Пользователи когорты 35-44 летних покупают реже, но в сумме объем их покупок примерно такой же.

Российские особенности ритейла выражаются, в том числе, в том, что большинство потребителей готовы переплачивать за скорость доставки и за доставку в назначенное время. Эту услугу особенно ценят покупатели старшей возрастной группы. Жители мегаполисов срочность доставки вообще относят к стандартным условиям оказания услуги.

Доставка еды и продуктов для её приготовления растёт особенно быстро вследствие сочетания растущего качества услуги и спектра удовлетворяемых ею потребностей и роста численности контингента потребителей. Наибольший интерес у потребителей вызывает доставка готовой еды - к ней относится до $80 \%$ поисковых запросов. Большие потенциалы роста имеет и доставка прочих товаров повседневного спроса. Для нас важно то, что эта услуга вошла в стадию саморазвития - она сама создаёт условия выработки у пользователей новых потребностей, связанных с доставкой заказов на дом.

На рынке труда курьерская доставка вошла в топ-3 наиболее популярных способов подработки, вместе с работой в такси и репетиторством. В то же время, наблюдаются признаки приближения к верхнему пределу количества курьеров на улицах. В частности, в Москве и Санкт-Петербурге перед властями уже стоит задача поиска консенсуса между интересами пешеходов, курьероввелосипедистов из сервисов доставки и автомобилистов.

При этом, по данным рекрутинговой компании HeadHunter, в России только за прошедшее лето почти втрое вырос спрос на курьеров - появилось свыше 25 тысяч вакансий. Из них Москве нужны 6 тыс. человек, Санкт-Петербургу - почти 3 тыс., а Московской области - 2 тыс.

Если по окончании пандемии спрос на услугу курьерской доставки и снизится, ясно, что у населения останется закрепившийся потребительский опыт, способствующий росту объёмов е-коммерции с доставкой.

Зарплаты сотрудников системы доставки. Центр стратегических разработок и Общероссийский народный фронт в мае сообщили, что в среднем курьер получает 400 руб. в час. Таким образом, доставщики стали лидерами по заработкам среди неквалифицированных сотрудников. Максимальный доход курьера на личной машине в Москве достигает 175 тыс. руб. в месяц. Велокурьера - 110 тыс., правда, при условии, что он ежедневно будет выполнять много заказов.

По данным HeadHunter, самая высокая медиана зарплаты курьерам - 67 тыс. руб. в месяц - сложилась в Московской области. В СанктПетербурге - 64,5 тыс. рублей.

Сложности создания доставки. По оценкам сервиса Bringo, построение собственной службы доставки для компании с несколькими десятками заказов в день обойдётся примерно в 1 млн рублей. Поэтому на старте предпочтительней обратиться в логистические компании, которые по запросу предоставляют нужное число курьеров.

Свидетельства ускорения. По свидетельству аналитиков BCS Global Markets, при том, что у крупных продуктовых ретейлеров доля онлайнвыручки пока не превышает 1-3\%, наблюдается тренд явного роста - 94\% покупателей после первого раза делают заказ на доставку повторно.

В "Яндекс.Маршрутизации" отмечают рост инвестиций в автоматизацию логистики, повышения квалификации персонала, поиск новых способов доставки и перепрофилирование простаивающих торговых помещений в дарксторы, максимально приближенные к жилым массивам.

Продукты из сетевых магазинов всё чаще доставляют курьеры логистических компаний, а производители продуктов выходят на маркетплейсы и напрямую контактируют с конечным потребителем.

По мере выявления и решения так называемых $\mathrm{UX}$-проблем в пользовательских интерфейсах систем е-коммерции, количество клиентов доставки потребительских товаров будет прогрессивно возрастать.

В отношении самого понятия робот-курьер отметим, что пандемия COVID-21 закрепила дополнительное звено - курьера в нисходящей цепочке поставок товаров «Поставщики материалов - Производство товаров Дистрибьютеры - Ритейлеры - Покупатели». При этом, вызванные пандемией новые потребности клиентов в бесконтактной доставке, полностью безналичном расчете, предоставлении сведений о состоянии здоровья курьера и о процессе осуществления доставки хаотизировали доставку последней мили. Соответственно, в удлинившейся цепочке поставки товаров усилился и стал чаще возникать эффект «хлыста» (Bullwhip-effect) c соответствующим увеличением издержек на всех высших звеньях.

Ещё одним важным обстоятельством является то, что в новых условиях проявились недостатки традиционной веерной доставка на «последней миле» людьми-курьерами из-за роста избыточных «холостых» маршрутов.

Состояние онлайн-ритейла в России. Российский рынок В2C e-commerce. Последние три года перед пандемией онлайн-торговля в 
России замедляла темпы роста, но карантинные ограничения повлекли многократный рост спроса со всеми вытекающими из этого последствиями. При этом объём онлайн-торговли рос незначительно из-за продолжившегося падения среднего чека покупки. Россия заняла шестое место в мире по объему В2С-торговли и её подстерегает опасность повторения американского «торгового апокалипсиса» из-за непомерного роста Amazon, сметающего более мелкие торговые сети. Пока ещё можно поддержать развитие «социальной коммерции», которая в Китае работает мощным сдерживающим фактором монополизации рынка еcommerce.

В целом, e-commerce в России может развиваться быстрыми темпами, поскольку бизнес охотно инвестирует, заработали обратные связи и стали раскрываться скрытые свободные ниши для технологий и сервисов электронной торговли. Но рост сдерживается снижением покупательской способности населения.

В настоящее время онлайн-гипермаркет «Vprok.ru Перекрёсток» обрабатывает до 15 тыс. заказов в день, а суммарное количество заказов во втором квартале 2021 года превысило 1 млн. при ежегодном росте на $13 \%$ и среднем чеке 4877 рублей. В России функционируют пять дарксторов «Vprok.ru Перекрёсток»: три в Москве (в Вешках и в Видном), один в Санкт-Петербурге и один в Нижнем Новгороде. В режиме курьерской доставки до двери заказчика водителями-экспедиторами на грузовиках «Vprok.ru Перекрёсток» работает в 8 регионах страны: в Москве, Санкт-Петербурге, а также Московской, Ленинградской, Нижегородской, Рязанской, Тульской и Тверской областях. Другие крупные участники ритейла также планируют активно развивать бизнес доставки последней мили [5]. Эксперты рынка считают нереализованными ещё резервами роста развитие онлайн-бизнеса в регионах, ожидаемый рост популярности новых групп товаров, неиспользованные площадки с развитой инфраструктурой и незначительные пока инвестиции основных игроков в рекламу.

По ожиданиям российских экспертов, постлокдауновское восстановление деловой активности должно поднять рынок онлайнторговли В2С до 2,4 трлн рублей и довести его долю до $6 \%$ от общего объема розничных продаж. А к 2024 году объем рынка e-commerce в России может составить 3,5 трлн руб.

Процессы на зарубежных рынках. В США и в Европе масштабы и уровень конкуренции за рынки побуждают повышенный интерес к проблемам и новациям в ритейле ${ }^{7} 89[6,7,8,9,10$, $11,12]$. Выводы авторов многочисленных

${ }^{7}$ Autonomous Last Mile Delivery Robots cut the highest cost of shipment // AGVnetwork. AGV and AMR Robot Home. https://www.agvnetwork.com/last-mile-delivery-robots

${ }^{8}$ Edwards D. Sidewalk last-mile delivery robots: Billiondollar market by 2030 // Robotics and Automation News. January 2020. https://roboticsandautomationnews.com/2020/01/02/sidewal исследований закрепляют в деловом сообществе давно сформировавшееся представление о безлюдной доставке как ключевом условии обеспечения будущего не только ритейлеров, электронной торговли, продуктовых сетей и ресторанов, но и непосредственно производственных компаний.

Ещё одним фактором, обусловливающим востребованность технологий безлюдной доставки, является приход в хозяйственную и культурную сферы жизнедеятельности поколения digital natives с их предпочтениями и навыками цифровизированных коммуникаций посредством разнообразных гаджетов. Для этого поколения потеряли актуальность экзистенциальные проблемы сосуществования обывателя с робототехническими системами. Более того, есть серьёзные предпосылки того, что в культурном коде бытования уже в последующие полтора-два десятилетия будут воспроизводится моральноэтические и правовые нормы сосуществования человека с неорганическими носителями интеллекта. Массовое внедрение безлюдной доставки явится первым шагом на этапе перехода к такому способу существования общества и индивида.

К наиболее значительным практическим шагам следует отнести запуск калифорнийского стартапа BoxBot с полностью автоматизированным сервисом доставки последней мили самоходными фургонами. Для тестирования своей технологии BoxBot объединился c компанией OnTrac, осуществляющей логистические услуги в западной части США для 65 млн клиентов. Согласно схеме доставки новым сервисом, рядом с жилыми районами будут располагаться автоматизированные локальные хабы для хранения и сортировки посылок, автоматически загружающихся в беспилотные фургоны для доставки с условием передвижения по проезжей части дорог.

По данным ReportCrux Market Research, среднегодовой рост рынка коммерческих дронов до 2027 года составит 24,3\%. Собственные программы развития доставки товаров дронами выполняют компании Amazon, Merck с доставками лекарств, Walmart в Лас-Вегасе с доставками тестов на COVID-19. Великобритания рассчитывает на экономию до £16 млрд с помощью внедрения технологий доставок дронами.

По результатам исследований The Boston Consulting Group в 2030 году общий объем мирового рынка робототехники может достичь 260 миллиардов долларов. При этом ожидается, что количество профессиональных сервисных роботов с объемом рынка от 90 до 170 миллиардов долларов

k-last-mile-delivery-robots-billion-dollar-market-by2030/28062/

${ }^{9}$ Nichols G. The last mile: Robots take to streets for local delivery // Zdnet. A red ventures company. March 2021. https://www.zdnet.com/article/the-last-mile-robots-take-tostreets-for-local-delivery/ 
намного превзойдёт количество обычных промышленных роботов и коботов.

Концептуальные подходы к роботизации доставки последней мили. Для ретейлеров ключевым ограничением инвестирования в роботизацию доставки на «последней миле» является представление о том, что невозможно сделать робота, который может съехать с гладкого асфальта и доставить заказ до двери заказчика. Действительно, проходимость представленных разработчиками наземных дронов не позволяет применять их для доставки товаров в городах с традиционным обустройством улиц и дорог.

Широкое использование воздушной доставки представляется нереалистичным. Например, в Москве уже сегодня работает до 35 тысяч курьеров ежедневно и требуется ещё 5-6 тысяч. Помимо того, что доставка воздушным дроном обходится на порядок дороже наземной доставки, горожане вряд ли согласятся с появлением в небе над городом большого количества жужжащих беспилотников.

Стартап с эстонскими корнями Starship Technologies продвигает на рынок шестиколёсных роботов-курьеров грузоподъёмностью до 18 кг. Эти экипажи в тестах преодолевают бордюры и невысокие лестницы. Такая проходимость достигнута за счёт применения балансирного шасси типа Rocker-bogie и адаптивного управления положениями осей шасси. Но и при таком существенном усложнении конструкции область применения этих роботов-курьеров ограничена участками с характерными для богатых одноэтажных городков Северной Америки инфраструктурными особенностями.

Практика попыток внедрения роботовкурьеров свидетельствует о том, что универсальные решения достижимы в виде дорогостоящих изменений обустройства среды обитания, или существенными изменениями дизайна самих роботов, или компромиссными решениями в обоих этих направлениях [13].

Очевидно, что направление переобустройством среды обитания предусматривает выполнение чрезвычайно дорогостоящих и долговременных программ, а наименее затратным и быстровыполнимым представляется изменение конструкции транспортной платформы роботов-курьеров.

Аналитические подходы к созданию безлюдных систем доставки. Многочисленные попытки аналитического описания процесса осуществления доставки последней мили предпринимаются давно $[14,15,16,17,18,19,20$, 21].

Эти работы посвящены, главным образом, оптимизации логистических схем при организации, управлении, планировании и контроле материальных и информационных потоков в пространстве и во времени в ходе осуществления цепочек доставки заказов в системе. Ядром этих задач являются задачи маршрутизации движения транспортных средств, которые, в свою очередь, сводятся к поиску решения «задачи коммивояжёра»
(TSP). TSP известна как NP-Hard проблема, для которой трудно найти оптимальное решение даже для случаев среднего размера входных параметров $[22,23,24,25,26,27]$. В простейшей постановке задача коммивояжёра сводится к нахождению последовательности обхода доставщиком заказчиков с минимальной общей протяжённостью маршрута. На практике эту задачу можно решить несколькими различными способами, каждый из которых является либо обобщением задачи коммивояжёра, либо использует её часть, вследствие чего все задачи маршрутизации следует считать NP-трудными для любых систем доставки.

Практика выполнения миссии коммивояжёра. В настоящее время коммивояжёры - курьеры-люди - обходят эти трудности, интуитивно используя «жадный алгоритм», дающий приемлемое, но не оптимальное решение, выполнимое за время, соразмерное длительности окна времени выполнения заказа. Эвристика «жадного» алгоритма заключается в выборе решения, являющегося лучшим в данный момент, независимо от того, улучшает или ухудшает этот выбор на следующем шаге.

Специфика действий людей-курьеров, управляющих транспортной машиной с механическим источником энергии, заключается в том, что они решают эвристические задачи, возникающие в виде изменений состояния системы местность-машина. Разнообразие форм таких изменений состояния и их динамика ограниченно специальными обустройствами трёхмерной среды их функционирования и конструкцией машины. Соответственно, только в пределах этих обустроенных областей пространства обеспечена управляемость машины и её полезность. Поэтому цепочки доставки многокомпонентные и обязательно включают элемент, предназначенный для перемещения товара в пространстве, обустроенном исключительно для пешего передвижения людей-курьеров, доставляющих товар «до двери».

Таким образом, роботизация доставки последней мили может быть сведена к роботизации двухзвенной цепочки доставки с использованием автодорожной транспортной техники в первом звене и высокомобильной автономной транспортной техники с подвижностью человека во втором звене. Первое - автотранспортное - звено роботизированной логистической цепочки доставки является традиционным объектом приложения усилий по роботизации. Вопросы роботизации этого звена тем или иным способом будут решены в ближайшее время. Второе звено предназначено для решения значительно более разнообразных алгоритмических, динамических и кинематических задач, возникающих в системе местность-доставщик в условиях перемещения по пространству, обустроенному под кинематические и динамические характеристики человека с его эвристическими способностями. 
Теоретическая база разработки систем управления робота-курьера. Разработчики системы управления для робота-курьера располагают теорией автоматического управления, оперирующей векторно-матричными моделями динамических систем. Теория позволяет моделировать многомерные системы со многими входами и выходами, описывая их векторноматричными уравнениями и аппаратом векторной алгебры. Описание модели с помощью системы дифференциальных уравнений первого порядка даёт пространство состояний в виде набора функций $x_{1}(t), x_{2}(t), \ldots, x_{n}(t)$, достаточно полно характеризующего модель системы, и наборы входных $u_{1}(t), u_{2}(t), \ldots, u_{r}(t) \quad$ и выходных $y_{1}(t), y_{2}(t), \ldots, y_{m}(t)$ параметров. Здесь $\mathrm{n}$ определяет размерность пространства состояний, r - размерность вектора входных параметров, а $\mathrm{m}$ размерность вектора выходных параметров.

Матрично-векторную модель динамической системы обычно представляют системой матричных уравнений, включающей уравнение состояния системы

$$
\dot{x}(t)=F[x(t), u(t), t],(1)
$$

и уравнение состояния выхода системы

$$
y(t)=Q[x(t), u(t), t]
$$

Здесь $\mathbf{F}-$ n-мерная вектор-функция состояния системы; Q - m-мерная векторфункция выхода.

Решением матричного уравнения состояния системы при начальном условии $\mathbf{x}_{0}=\mathbf{x}\left(\mathrm{t}_{0}\right)$ является вектор состояния системы

$$
\mathbf{X}(\mathrm{t})=\Psi\left[\mathbf{x}\left(\mathrm{t}_{0}\right), \mathrm{u}(\mathrm{t}), \mathrm{t}\right] .(3)
$$

Если известны начальное состояние системы (1), (2) при $\mathbf{t}=0$ и зависимость входных параметров модели от времени, решение (3) системы уравнения даст однозначное описание состояния модели в заданный момент времени.

В случае стационарности модели системы становится возможным оперирование со стационарными уравнениями

$$
\begin{aligned}
& \dot{\mathrm{x}}=\mathrm{Ax}+\mathrm{Bu},(4) \\
& \mathrm{y}=\mathrm{Cx}+\mathrm{Du},(5)
\end{aligned}
$$

где:

А - матрица состояния системы в виде функциональной матрицы размером $\boldsymbol{n} \times \boldsymbol{n}$, где $\boldsymbol{n}-$ количество переменных по времени независимых элементов системы;

В - матрица управления в виде функциональной матрицы размером $\boldsymbol{n} \times \boldsymbol{r}$, где $\boldsymbol{r}-$ количество независимых параметров управления;

C - матрица выхода по состоянию в виде функциональной матрицы размером $\boldsymbol{m} \times \boldsymbol{n}$, где $\boldsymbol{m}$
- количество независимых выходных параметров системы;

D - матрица выхода по управлению в виде функциональной матрицы размером $\boldsymbol{m} \times \boldsymbol{r}$;

В большинстве случаев зависимостью выхода от входа можно пренебречь, то есть, можно принять $\mathbf{D}=\mathbf{0}$.

Подобная единая форма представления позволяет алгоритмизировать компьютерную реализацию синтеза систем управления роботами в приближении линеаризации поведения реальных динамических систем.

Насколько применим этот аппарат в реальности, можно оценить на примерах с осуществления доставки различными способами.

Люди-курьеры в автомобиле, на мотоциклах, скутерах, велосипедах и пешие вынуждены решать всё большие по объёму эвристические задачи, поскольку размерность пространства состояний системы местность-мотоциклист существенно выше размерности пространства состояний системы местность-автомобиль, размерность пространства состояний системы местностьвелосипедист выше размерности пространства системы местность-мотоциклист, размерность пространства состояний системы местностьсамокатчик/моноциклист/гироскутерист выше размерности пространства состояний системы местность-велосипедист и, наконец размерность состояний системы местность-пешеход выше размерности пространства состояний системы местность-

самокатчик/моноциклист/гироскутерист.

Очевидно, что способность транспортного средства парировать изменения состояния системы местность-машина определяется массой машины и количеством связей в системе местность-машина.

Также очевидно, что эффективность машины достигается при условии её полной управляемости - способности передвижения по заданной траектории с заданной скоростью. Что, в свою очередь, достигается при оптимальном количестве связей в системе местность-машина в данных условиях эксплуатации, когда снижение энергоэффективности и управляемости из-за избыточных связей соразмерно выигрышу в способности парирования возникающих при движении машины по заданному маршруту воздействий.

Количество избыточных связей в системе местность-доставщик можно определить по известной формуле Сомова-Малышева:

$$
\mathrm{Q}=\mathrm{W}-6 \mathrm{n}+5 \mathrm{p}_{5}+4 \mathrm{p}_{4}+3 \mathrm{p}_{3}+2 \mathrm{p}_{2}+\mathrm{p}_{1}
$$

где Q - число степеней свободы с избыточными связями в системе, W - число степеней свободы системы местность-машина, $\mathbf{n}-$ количество звеньев в системе; $\mathbf{p}_{\mathbf{i}}-$ число звеньев системы местность-машина с і связями.

В частности, в системе местность-доставщик на базе автомобиля с наиболее распространённой колёсной формулой автомобиля $\mathbf{4 \times 2 \times 2}$ с 
независимой подвеской колёс число избыточных связей $\mathbf{Q}$ будет равно $5-30+25+20+6=26$.

А в системе местность-доставщик с человеком на самокате $\mathbf{Q}$ будет равно $3-24+5+4+2+5=$ 5. То есть, человеку-курьеру на самокате нехватает 5 связей для обеспечения статической уравновешенности. Соответственно, для поддержания устойчивости он подавляет 5 избыточных подвижностей в системе местностьсамокат, создавая своим телом управляющие силы и моменты.

Пеший курьер в механическом смысле представляет собой статически неуравновешенную систему, поддерживающую равновесие посредством управления динамической остойчивостью, известного как управление остойчивостью обратного физического маятника. Исходя из известного количества степеней свободы человеческого тела примерно $6 \times 144$ - $5 \times 81$ - 4 × $33-3 \times 29=240$, для системы местность - курьерчеловек получим $\mathbf{Q} \sim 234-84+15=165$ избыточных подвижностей.

Очевидно, что попытка замещения курьерачеловека антропоморфным курьером-роботом столкнётся с проблемами наложения около 165 дополнительных связей на избыточные подвижности в системе местность - робот-курьер.

Соответственно, поскольку для решения системы уравнений (5), (6) следует использовать входные данные в количестве, близком к количеству действующих связей в системе местность-курьер, разработка эффективной системы управления движением робота-курьера, обеспечивающей необходимую для доставки «до двери» управляемость, потребует использования сложных быстродействующих вычислительных систем со всеми сопутствующими обеспечивающими устройствами и источниками энергии на работу и охлаждение.

Иными словами, разработки роботовдоставщиков последней мили в виде антропоморфных или, в пределе, антропомиметических робототехнических агрегатов представляются иррациональными с точки зрения достижения полезного эффекта от роботизации доставки.

Перспективы применения иных транспортных платформ следует рассмотреть в отдельном исследовании.

\section{Литература:}

1.Latest Global Outlook - October/November 2021 Global Business Pulse // Published by Oxford Economics. 2021. [Электронный pecypc]. https://www.oxfordeconomics.com/

2.World Drone Market Seen Nearing \$127 Billion in 2020, PwC Says // Bloomberg.com. May 2017.

3.Колесов К.И. Финансирование инновационных проектов: учебное пособие // Н. Новгород. Издательство Нижегородского государственного технического университета им P.Е. Алексеева. 2017. С. 118. [Электронный ресурс].
https://citr.nntu.ru/upload/FCfinder/files/izdaniya/fina ns_innov_proect.pdf

4.Исследование ценностей миллениалов в 2020 году в России // Ежегодный отчет Deloitte Millennial Survey 2020. [Электронный pecypc]. https://www2.deloitte.com/ru/ru/pages/aboutdeloitte/articles/millennial-survey-2020.html

5.Deloitte University Press. The future of freight. How new technology and new thinking can transform how goods are moved. Deloitte Center for Integrated Research. 2017. 30 p. [Electronic resource]. https://www2.deloitte.com/insights/us/en/focus/futureof-mobility/future-of-freight-simplifying-last-milelogistics.html

6.Маркетинговое исследование и анализ российского рынка услуг экспресс-доставки // МА Step by step. Август 2018. С.16. [Электронный pecypc]. http://www.step-bystep.ru/upload/iblock/907/ demo_dostavka.pdf

7.Joerss M., Schröder J., Neuhaus F., Klink C., Mann F. Parcel delivery The future of last mile // McKinsey \& Company. September 2016. [Electronic resource].

https://www.mckinsey.com/ /media/mckinsey/industr ies/travel\%20transport\%20and\%20logistics/our\%20in sights/how\%20customer\%20demands $\% 20$ are $\% 20$ resh aping\%20last\%20mile\%20delivery/parcel_delivery_th e_future_of_last_mile.ashx

8.The Future of the Last-Mile Ecosystem // World Economic Forum 91-93 route de la Capite CH-1223 Cologny. Geneva. Switzerland. January 2020. [Electronic resource]. https://www3.weforum.org/docs/WEF_Future_of_the _last_mile_ecosystem.pdf

9.Wang X., Zhan L., Ruan J., Zhang J. How to Choose "Last Mile" Delivery Modes for EFulfillment.// Mathematical Problems in Engineering. V. 2014. Jun 2014. [Electronic resource]. https://doi.org/10.1155/2014/417129

https://www.hindawi.com/journals/mpe/2014/417129/

?utm_source=google\&utm_medium $=$ cpc\&utm_campa ign=HDW_MRKT_GBL_SUB_ADWO_PAI_DYNA _JOUR_X\&gclid=Cj0KCQjwiNSLBhCPARIsAKNS 4_dIC697agDngLp9jWe_6WSX37Cr1Wy1dmLvsi3J 05wIaf7wEnEnlqAaAr3UEALw_wcB

10.Caccamo S. Last Mile Delivery Robots // Univrses journal. August 2020. [Electronic resource]. https://univrses.com/case-study/last-mile-deliveryrobots-new/

11.Левкин Г., Скаков Р. Что поправить на последней миле - примеры «Леруа Мерлен», «Деловых линий» // Издательский дом «Гребенников». Март 2021. [Электронный ресурс]. https://pr.dellin.ru/smi/chto-popravit-na-poslednejmile-primery-lerua-merlen-delovyh-linij/

12.Bouvet N., Kaltenbach P. Reroute your strategy for last - mile delivery // Accenture strategy. Accenture Adaptive Retail Survey. February 2017. 10 p. [Электронный ресурс].

https://www.accenture.com/t00010101T000000Z__w

_/fr-fr/_acnmedia/PDF-62/Accenture-Last-Mile-

POV.pdf

13.Мочалин C.M. Математическая модель 
описания транспортного процесса в средних системах доставки грузов // Вестник ОГУ. 2004. №2. C. $185-189$.

14. Горев А. Э. Основы теории транспортных систем // Издательство Санкт-Петербургского государственного архитектурно-строительного университета. 2010. С. 214.

15.Иванов Д. А. Управление цепями поставок // Издательство Политехнического университета. 2009. С. 660.

16.Пустохина И. Управление цепями поставок: проблемы, их причины и пути решения // Логистика. 2013. №10. С. 35-37.

17.Тюкина Л. В. Анализ параметров заявки на доставку грузов автомобильным транспортом // Омск: Сборник научных трудов молодых ученых по материалам Международной научнопрактической конференции Инновационное лидерство строительной и транспортной отрасли глазами молодых ученых. 2014. С. 352-357.

18.Лукинский В.С., Бережной В. И., Бережная Е. В. и др. Логистика автомобильного транспорта // Финансы и статистика. 2004. С. 368.

19.Jinghong W., Yuanyuan H. Optimization of the "last mile" operation mode of e-commerce express delivery // Modern Business and Industry. 2018. Pp. 34-35.

20.Zhang Y., Fan X., Zhou L. Analysis and Research on the "last mile" distribution innovation model of e-commerce express delivery // Journal of Physics: Conf. Series 1176. 2019. doi:10.1088/17426596/1176/4/042044 [Electronic resource]. https://iopscience.iop.org/article/10.1088/17426596/1176/4/042044/pdf

21.Qiran H., Guangxian W. Research on the Receiving Model of the Last Kilometer of E-commerce Distribution // Journal of Jiaying University. 2014. 32(04) p. 37-41.

22.Wang J. Research on the "last mile" logistics distribution model in e-commerce environment // Beijing Jiaotong University. 2017.

23.Льюис Г., Пападимитриу Х. Элементы теории вычислений // Недетерминированные машины Тьюринга. Раздел 4.6 (1-е изд.). ПрентисХолл. 1981. С. 204-211.

24.Гэри М., Джонсон Д. Вычислительные машины и труднорешаемые задачи // Монография. Издательство «Мир». 1982. С. 411

25.Джон К. М. Введение в языки и теорию вычислений // Недетерминированные машины Тьюринга. Раздел 9.6. (2-е изд.). Макгроу-Хилл. 1997. С. 277-281.

26.Пападимитриу X. Вычислительная сложность // Недетерминированные машины. Раздел 2.7. (1-е изд.). Эддисон-Уэсли. 1993. С. 45-50.

27.Стивен В. Новый вид науки // Wolfram Media. Inc. 2002. C. 967-968.

\section{Literature:}

1.Latest Global Outlook - October/November 2021 Global Business Pulse // Published by Oxford Economics. [Electronic resource]. https://www.oxfordeconomics.com/
2.World Drone Market Seen Nearing \$ 127 Billion in 2020, PwC Says // Bloomberg.com. May 2017

3.Kolesov K.I. Financing innovative projects: a tutorial // N. Novgorod. Publishing house of the Nizhny Novgorod State Technical University named after R.E. Alekseeva. 2017. 118 p. [Electronic resource]. https://citr.nntu.ru/upload/FCfinder/files/izdaniya/fina ns_innov_proect.pdf

4.Marketing research and analysis of the Russian market for express delivery services // MA Step by step. August 2018. 16 p. [Electronic resource]. http://www.step-by-step.ru/upload/iblock/907/ demo_dostavka.pdf

5.The impact of the express delivery industry on the global economy // Oxford Economics. September 2009. 48. p. [Electronic resource]. https://www.oxfordeconomics.com/publication/downl oad/228797

6.Reaching the Last Mile: Challenges and Opportunities // Global Washington. March 2016. [Electronic resource]. https://globalwa.org/issuebrief/last-mile-delivery/

7.Joerss M., Schröder J., Neuhaus F., Klink C., Mann F. Parcel delivery The future of last mile // McKinsey \& Company. September 2016. [Electronic resource]

https://www.mckinsey.com/ /media/mckinsey/industr ies/travel\%20transport\%20and\%20logistics/our\%20in sights/how\%20customer\%20demands $\% 20$ are $\% 20$ resh aping\%20last\%20mile\%20delivery/parcel_delivery_th e_future_of_last_mile.ashx

8.The Future of the Last-Mile Ecosystem // World Economic Forum 91-93 route de la Capite CH-1223 Cologny. Geneva. Switzerland. January 2020. [Electronic resource]. https://www3.weforum.org/docs/WEF_Future_of_the _last_mile_ecosystem.pdf

9.Wang X., Zhan L., Ruan J., Zhang J. How to Choose "Last Mile" Delivery Modes for E-Fulfillment // Mathematical Problems in Engineering. V. 2014. Jun 2014. [Electronic resource]. https://doi.org/10.1155/2014/417129

10.Caccamo S. Last Mile Delivery Robots.// Univrses journal. August 2020. [Electronic resource]. https://univrses.com/case-study/last-mile-deliveryrobots-new/

11.Levkin G., Skakov R. What to fix on the last mile - examples of "Leroy Merlin", "Business lines" // Publishing house "Grebennikov". March 2021. [Electronic resource]. https://pr.dellin.ru/smi/chtopopravit-na-poslednej-mile-primery-lerua-merlendelovyh-linij/

12.Bouvet N., Kaltenbach P. Reroute your strategy for last - mile delivery // Accenture strategy. Accenture Adaptive Retail Survey. February 2017. 10 p. [Electronic resource]. https://www.accenture.com/t00010101T000000Z_w _/fr-fr/_acnmedia/PDF-62/Accenture-Last-MilePOV.pdf

13.Mochalin S.M. Mathematical model for describing the transport process in medium-sized cargo delivery systems // Vestnik OSU. 2004. No. 2. Pp. 185 - 189. 
14.Gorev A. E. Fundamentals of the theory of transport systems // St. Petersburg: Publishing house of the St. Petersburg State University of Architecture and Civil Engineering, 2010. 214 p.

15.Ivanov D. A. Supply Chain Management // St. Petersburg: Publishing House of the Polytechnic University. 2009. 660 p.

16.Pustokhina I. Supply chain management: problems, their causes and solutions. // Logistics. 2013. No. 10. Pp. 35-37.

17.Tyukina L.V. Analysis of the parameters of the application for the delivery of goods by road // Omsk: Collection of scientific works of young scientists based on the materials of the International Scientific and Practical Conference Innovative leadership of the construction and transport industry through the eyes of young scientists. 2014. Pp. 352-357.

18.Lukinsky V.S., Berezhnoy V. I., Berezhnaya E. V. et al. Logistics of motor transport // Finance and statistics. 2004. 368 p.

19.Jinghong W., Yuanyuan H. Optimization of the "last mile" operation mode of e-commerce express delivery // Modern Business and Industry. 2018. Pp. 34-35.

20.Zhang Y., Fan X., Zhou L. Analysis and Research on the "last mile" distribution innovation model of e-commerce express delivery // Journal of
Physics: Conf. Series 1176. 2019. doi:10.1088/17426596/1176/4/042044 [Electronic resource]. https://iopscience.iop.org/article/10.1088/17426596/1176/4/042044/pdf

21. Qiran H., Guangxian W. Research on the Receiving Model of the Last Kilometer of E-commerce Distribution // Journal of Jiaying University. 2014. 32(04) Pp. 37-41.

22.Wang J. Research on the "last mile" logistics distribution model in e-commerce environment // Beijing Jiaotong University. 2017.

23.Lewis G., Papadimitriou H. Elements of the theory of computation // Non-deterministic Turing machines. Section 4.6 (1st ed.). Prentice Hall. 1981. Pp. 204-211.

24.Gary M., Johnson D. Computing machines and intractable problems // Monograph. Publishing house "Mir". 1982. 411 p.

25.John K. M. Introduction to languages and computation theory // Non-deterministic Turing machines. Section 9.6 (2nd ed.). McGraw Hill. 1997. Pp. 277-281.

26.Papadimitriou H. Computational complexity // Non-deterministic machines. Section 2.7 (1st ed.). Addison-Wesley. 1993. Pp. 45-50.

27.Stephen V. A new kind of science // Wolfram Media. Inc. 2002. Pp. 967-968. 